\title{
BMJ Open Rationale and design of Short-Term EXenatide therapy in Acute ischaemic Stroke (STEXAS): a randomised, open-label, parallel-group study
}

\author{
Rachel T McGrath, ${ }^{1,2,3}$ Samantha L Hocking, ${ }^{1,2}$ Miriam Priglinger, ${ }^{4}$ Susan Day, ${ }^{4}$ \\ Geoffrey K Herkes, ${ }^{2,4}$ Martin Krause, ${ }^{2,4}$ Gregory R Fulcher ${ }^{1,2}$
}

To cite: McGrath RT, Hocking SL, Priglinger M, et al. Rationale and design of Short-Term EXenatide therapy in Acute ischaemic Stroke (STEXAS): a randomised, open-label, parallel-group study. BMJ Open 2016;6: e008203. doi:10.1136/ bmjopen-2015-008203

- Prepublication history and additional material is available. To view please visit the journal (http://dx.doi.org/ 10.1136/bmjopen-2015008203).

Received 15 March 2015 Revised 19 August 2015 Accepted 21 September 2015

\section{CrossMark}

For numbered affiliations see end of article.

\section{Correspondence to} Dr Rachel McGrath; Rachel.McGrath@health.nsw. gov.au

\section{ABSTRACT \\ Introduction: Both hyperglycaemia and} hypoglycaemia in acute ischaemic stroke (AIS) are associated with increased infarct size and worse functional outcomes. Thus, therapies that can maintain normoglycaemia during stroke are clinically important. Glucagon-like peptide 1 (GLP-1) analogues, including exenatide, are routinely used in the treatment of hyperglycaemia in type 2 diabetes, but data on the usefulness of this class of agents in the management of elevated glucose levels in AIS are limited. Owing to their glucose-dependent mechanism of action, GLP-1 analogues are associated with a low risk of hypoglycaemia, which may give them an advantage over intensive insulin therapy in the acute management of hyperglycaemia in this setting.

Methods and analysis: The Short-Term EXenatide therapy in Acute ischaemic Stroke study is a randomised, open-label, parallel-group pilot study designed to investigate the efficacy of exenatide at lowering blood glucose levels in patients with hyperglycaemia with AIS. A total of 30 patients presenting with AIS and blood glucose levels $>10 \mathrm{mmol} / \mathrm{L}$ will be randomised to receive the standard therapy (intravenous insulin) or intravenous exenatide for up to $72 \mathrm{~h}$. Outcomes including blood glucose levels within the target range $(5-10 \mathrm{mmol} / \mathrm{L})$, the incidence of hypoglycaemia and the feasibility of administering intravenous exenatide in this patient population will be assessed. A follow-up visit at 3 months will facilitate evaluation of neurological outcomes post-stroke.

Ethics and dissemination: This study has been approved by the local Institutional Review Board (Northern Sydney Local Health District Human Research Ethics Committee). The study results will be communicated via presentations at scientific conferences and through publication in peer-reviewed journals.

Conclusions: As GLP-1 analogues require elevated glucose levels to exert their insulin potentiating activity, the use of exenatide in the management of hyperglycaemia in AIS may reduce the incidence of hypoglycaemia, thereby conferring a benefit in morbidity and mortality for patients in the long term.

Trial registration number: ACTRN12614001189617.

\section{Strengths and limitations of this study}

- Owing to the mechanism of action of exenatide it is envisaged that treatment with exenatide will result in a lower rate of hypoglycaemia, thereby improving outcomes for patients with acute ischaemic stroke.

- Another strength of this study is that we are using continuous glucose monitoring to measure glycaemic control, which will provide detailed information about the incidence of hyperglycaemia and hypoglycaemia, as well as glycaemic variability.

- A limitation of this study is that it is not powered to determine functional outcomes for patients post-stroke. However, by demonstrating that exenatide treatment in this setting is safe and feasible, useful preliminary data will be generated that can be used to design larger clinical studies.

\section{BACKGROUND}

The association between diabetes mellitus and an increased risk of vascular events, including acute ischaemic stroke (AIS), is well documented. Elevated blood glucose levels are frequently observed in patients with AIS, and correlate with a poor clinical outcome. ${ }^{12}$ Of note, the negative effects of hyperglycaemia in AIS have been shown for both individuals with diabetes and without diabetes. ${ }^{3}{ }^{4}$ Elevated blood glucose levels are seen in up to $50 \%$ of patients without diabetes with AIS, and are associated with a significantly worse functional outcome, as well as a threefold increased risk of both inhospital and long-term mortality. ${ }^{4}$ Further evidence has demonstrated bigger infarct size as well as expansion of infarcts in hyperglycaemic patients, and an accelerated loss of penumbral tissue when high blood glucose levels are present. ${ }^{5}$ The high blood glucose levels in patients not known to have diabetes 
who present with AIS may indicate undiagnosed diabetes, insulin resistance, or impaired glucose tolerance. Also, stress-induced hyperglycaemia may occur, where release of glucocorticoids and catecholamines leads to impairment of insulin secretion and stimulation of hepatic glucose production. ${ }^{67}$

The detrimental effect of hyperglycaemia on functional outcome after cerebral ischaemia can be reversed through administration of insulin and lowering of blood glucose levels. Current guidelines in Europe (European Stroke Association) and the USA (American Heart Association/ American Stroke Association) recommend management of hyperglycaemia in the setting of AIS by initiation of insulin therapy when blood glucose levels are above $10 \mathrm{mmol} / \mathrm{L}^{8}{ }^{9}$ Intensive insulin treatment (IIT) with intravenous insulin infusion can normalise glycaemia, and there is some evidence suggesting it improves functional outcomes in AIS. ${ }^{10}{ }^{11}$ However, IIT is associated with hypoglycaemia, which adversely affects outcome and increases mortality and morbidity. ${ }^{12}$ In addition, IIT is frequently administered using a sliding scale, which is difficult to implement and both time-intensive and labour-intensive for treating clinical staff. In the intensive care setting, for both medical and surgical patients, the incidence of hypoglycaemia was significantly higher when intensive insulin therapy was used, and insulin-treated patients with hypoglycaemia exhibited higher mortality rates in comparison to non-insulin-treated controls. ${ }^{13-15}$

A systematic review published in 2014 gave an overview of clinical trials in which insulin was administered to treat hyperglycaemia in AIS, and concluded that administration of intravenous insulin does not result in a benefit in terms of functional outcome or mortality and significantly increased hypoglycaemia. ${ }^{16}$ Similarly, the glucose insulin in stroke trial study demonstrated that insulin infusion in mild to moderate hyperglycaemia in AIS effectively lowers blood glucose levels but does not give rise to improvements in clinical outcomes. Furthermore, in the insulin-treated arm, hypoglycaemia requiring rescue treatment with intravenous glucose was observed in approximately $16 \%$ of patients. ${ }^{17}$ Evidence surrounding the impact of hypoglycaemia in AIS is scant, however, one study has shown an association between blood glucose levels below $3.7 \mathrm{mmol} / \mathrm{L}$ and a poorer functional outcome. ${ }^{18}$

An alternate approach to glycaemic management in AIS is the use of glucagon-like peptide 1 (GLP-1) analogues. GLP-1 is an incretin hormone secreted from the gut in response to nutrient intake, which potentiates insulin secretion from pancreatic $\beta$ cells in a glucosedependent manner. ${ }^{19}$ GLP-1 analogues, such as exenatide, are routinely used in the management of type 2 diabetes. Furthermore, due to their glucose-dependent mechanism of action, GLP-1 analogues as monotherapy are associated with a minimal risk of hypoglycaemia. ${ }^{20}$

Several preclinical studies have demonstrated that treatment with GLP-1 reduces blood glucose levels and leads to improved functional outcomes in rodent models of type 2 diabetes and acute stroke. ${ }^{21} \quad 22$ Furthermore, GLP-1 can act directly on the brain to reduce cerebral hyperglycaemia, thereby decreasing oxidative stress and neuronal cell apoptosis. ${ }^{23}{ }^{24}$ This neuroprotective activity of GLP-1 can occur independently of its glucose-lowering activity, as the GLP-1 receptor is widely distributed throughout the central nervous system, and direct administration of GLP-1 to the brain of mice showed improvements in ischaemia-related inflammation and apoptosis. ${ }^{22}$

Recently, it was demonstrated that subcutaneous administration of exenatide effectively lowered blood glucose levels in patients with AIS, with minimal incidence of hypoglycaemia and reduced glycaemic variability. ${ }^{25}$ In addition, in the presence of hypoglycaemia, GLP-1 does not reduce brain glucose concentration, nor does it influence glucose metabolism, or transfer across the blood-brain barrier. ${ }^{26}$ Thus, GLP-1 may be a more suitable therapeutic strategy for maintaining normoglycaemia in the ischaemic brain, with less variability in blood glucose levels occurring over the treatment period.

This pilot study has been designed to investigate whether the GLP-1 analogue exenatide is a useful therapeutic option for the treatment of hyperglycaemia during AIS. Promising preliminary studies and preclinical data suggest that GLP-1 will provide effective glycaemic control while minimising the risk of hypoglycaemia, thereby reducing adverse outcomes that may be experienced with standard insulin therapy. This may give rise to clinical benefits for patients post-stroke. We propose that GLP-1 will demonstrate superiority in comparison to insulin, at minimising the frequency and duration of hypoglycaemia, and will be as efficacious as insulin at regulating blood glucose levels and attaining glycaemic targets, in AIS.

\section{METHODS/DESIGN}

\section{Study overview}

The STEXAS (Short-Term EXenatide therapy in Acute ischaemic Stroke) study is a randomised, open-label, parallel-group pilot study in which the efficacy of exenatide at lowering blood glucose levels in patients with hyperglycaemia and AIS will be evaluated. In addition, the safety and tolerability of exenatide treatment in this setting will be determined. A total of 30 patients will be recruited and randomised to receive either the standard therapy for hyperglycaemia in AIS, that is, insulin, or exenatide. Both insulin and exenatide will be administered intravenously for up to $72 \mathrm{~h}$, consistent with current therapeutic guidelines in AIS. To be eligible for participation in the study, patients must meet the study inclusion and exclusion criteria, outlined in box 1. This study is investigator-initiated and the local study sponsor is the Northern Sydney Local Health District.

\section{Study population, enrolment and randomisation}

Men and women aged $<18$ years, admitted to the Acute Stroke Ward in Royal North Shore Hospital, Sydney, with 


\section{Box 1 Eligibility criteria}

\section{Inclusion criteria}

- Clinically defined acute ischaemic stroke with consistent neuroimaging (eg, CT brain, CT perfusion or MRI brain)

- Blood glucose levels $>10 \mathrm{mmol} / \mathrm{L}$ on blood glucose testing during the first $72 \mathrm{~h}$ after admission to the acute stroke ward

- Age $>18$ years

Exclusion criteria

- Type 1 diabetes mellitus

- Initial National Institutes of Health Stroke Scale $>20$

- Life expectancy below 12 months

- Premorbid Modified Rankin Score above 3

- Impaired liver function, defined as alanine aminotransferase $(A L T) \geq 3$ times the upper limit of normal

- Impaired renal function, defined as an estimated glomerular filtration rate $<30 \mathrm{~mL} / \mathrm{min} / 1.73 \mathrm{~m}^{2}$

- History of pancreatitis

- History of pancreatic cancer

- History of medullary thyroid cancer or multiple endocrine neoplasia II

Known allergy to exenatide

Suspected or known abuse of alcohol or narcotics

Pregnancy or lactation

acute neurological deficit of suspected vascular origin and neuroimaging (CT brain, CT perfusion or MRI) evidence of AIS, with a blood glucose level of $<10 \mathrm{mmol} / \mathrm{L}$ within $72 \mathrm{~h}$ after admission are eligible to take part in the study. Blood glucose levels will be determined by standard laboratory analysis and by finger-prick capillary glucose testing using a blood glucose meter. Written informed consent will be obtained from all participants, prior to study procedures taking place. In the instance that a potential study participant is unable to give written informed consent, written informed assent from their guardian will be obtained. Only patients with persistent neurological deficits caused by their stroke at study entry will be included.

On study entry, participants will be randomised to receive either intravenous insulin or intravenous exenatide for the duration of the study (up to $72 \mathrm{~h}$, or until discharge from the acute stroke ward). Block randomisation will be used, such that balance across the two treatment groups is achieved. On-site randomisation will be carried out using light-impermeable envelopes containing study arm allocation. In addition, participants will be stratified, based on their current use of insulin, such that there is equal distribution of patients that take insulin (prior to study entry) across the treatment arms. Study researchers and clinicians will be unblinded to the treatment arm allocation, due to the differences in the treatment protocol for exenatide versus insulin infusion.

\section{Study intervention}

Study participants allocated to the exenatide treatment arm will receive continuous intravenous administration of $20 \mu \mathrm{g} / 24 \mathrm{~h}$ of exenatide (Byetta; Bristol-Myers
Squibb/AstraZeneca, Australia) diluted in saline containing $0.2 \%$ human serum albumin. ${ }^{27}{ }^{28}$ Then, $0.2 \mathrm{~mL}$ of exenatide (drawn from a $5 \mu \mathrm{g}$ Byetta pen containing $0.25 \mathrm{mg}$ exenatide $/ \mathrm{mL}$ ) will be added to a $250 \mathrm{~mL}$ saline bag containing $2.5 \mathrm{~mL}(0.2 \%)$ of Albumex 20 (CSL Limited, Australia). Exenatide will be infused at a rate of $4.2 \mathrm{~mL} / \mathrm{h}$ and a new preparation of exenatide for intravenous administration will be made up every $24 \mathrm{~h}$. In total, patients allocated to the exenatide treatment arm will receive $60 \mu \mathrm{g}$ of exenatide over 3 days, that is, $20 \mu \mathrm{g}$ of exenatide per $24 \mathrm{~h}$. The intravenous route was chosen such that therapeutic exenatide activity will be achieved within a short time frame and maintained over the course of the study treatment period. ${ }^{29} \quad 30$ Furthermore, the dose of exenatide will not be up-titrated over time (as is the standard practice with subcutaneous exenatide) to ensure that optimum glycaemic control will be attained as rapidly as possible.

Participants allocated to the insulin treatment arm will receive an intravenous insulin infusion (with indicated number of units of fast-acting insulin (Act Rapid, Novo Nordisk) diluted in saline and concomitant $5 \%$ glucose as per local hospital protocol).

In both arms of the study, oral diabetes medications will be withheld for the duration of the exenatide/ insulin infusion (in keeping with the local hospital's insulin infusion protocol).

For the exenatide arm, if blood glucose levels remain higher than $12 \mathrm{mmol} / \mathrm{L}$ for longer than $3 \mathrm{~h}$, intravenous insulin infusion will be added as a rescue therapy, which will continue up until the end of the study, that is, the $72 \mathrm{~h}$ time point. Nausea is a common side effect of GLP-1 analogue therapy when given subcutaneously, due to the effect of GLP-1 on reducing the rate of gastric emptying. Relatively little is known about the gastrointestinal (GI) side effects of exenatide when administered intravenously, however, recent evidence suggests that intravenous administration produces less nausea than subcutaneous administration. ${ }^{31}$ Moreover, the rationale for dose titration with subcutaneous exenatide therapy is related to GI side effects that are not likely to be an issue with intravenous administration.

If a participant experiences significant nausea while receiving an exenatide infusion, they will be treated with metoclopramide, according to standard clinical guidelines. If the nausea does not decrease on treatment with metoclopramide, the rate of exenatide infusion will be reduced by $50 \%$, until resolution of nausea. If significant nausea fails to resolve within $3 \mathrm{~h}$, exenatide treatment will be stopped and insulin rescue therapy started.

\section{Study assessments}

Study assessments (table 1) comprise evaluation of glycaemic control by hourly capillary blood glucose measurements using the Optium Xceed glucose meter (Abbott, Australia) and blinded continuous glucose monitoring (iPro Continuous Glucose Monitoring System; Medtronic, Australia). Neurological assessments 
Table 1 Overview and timing of study procedures and assessments

\begin{tabular}{|c|c|c|c|c|c|}
\hline & $\begin{array}{l}\text { Day } 0 \text { screening/ } \\
\text { baseline }\end{array}$ & $\begin{array}{l}\text { 0-24 h } \\
\text { post-stroke }\end{array}$ & $\begin{array}{l}24-48 \mathrm{~h} \\
\text { post-stroke }\end{array}$ & $\begin{array}{l}48-72 \mathrm{~h} \\
\text { post-stroke }\end{array}$ & $\begin{array}{l}3 \text { Months } \\
\text { post-stroke }\end{array}$ \\
\hline Inclusion/exclusion criteria & $\mathrm{X}$ & & & & \\
\hline Informed consent & $x$ & & & & \\
\hline Medical history & $x$ & & & & $\mathrm{X}$ \\
\hline Randomisation & $x$ & & & & \\
\hline $\begin{array}{l}\text { Glucagon-like peptide } 1 \text { RA/ } \\
\text { insulin infusion }\end{array}$ & & $x$ & $x$ & $x$ & \\
\hline $\begin{array}{l}\text { National Institutes of Health } \\
\text { Stroke Scale (NIHSS) }\end{array}$ & $x$ & $x$ & & $x$ & $x$ \\
\hline Modified Rankin scale & X (premorbid) & & & & $\mathrm{X}$ \\
\hline CT brain & $x$ & & & & \\
\hline $\begin{array}{l}\text { Blood glucose level } \\
\text { (laboratory) }\end{array}$ & $x$ & $\mathrm{X}$ & $x$ & $x$ & $x$ \\
\hline $\begin{array}{l}\text { Blood glucose level } \\
\text { (finger-prick test) }\end{array}$ & $x$ & & & & \\
\hline Hourly blood glucose level & & $x$ & $\mathrm{X}$ & $\mathrm{X}$ & \\
\hline Blood collection (research) & $\mathrm{X}$ & $\mathrm{X}$ & $\mathrm{X}$ & $\mathrm{X}$ & \\
\hline Frequency of hypoglycaemia & $x$ & $\mathrm{X}$ & $\mathrm{X}$ & $x$ & \\
\hline Collection of adverse events & $\mathrm{X}$ & $x$ & $x$ & $\mathrm{X}$ & $\mathrm{X}$ \\
\hline
\end{tabular}

with the National Institutes of Health Stroke Scale (NIHSS) will be performed at baseline, 24 and $72 \mathrm{~h}$ and 3 months postinitiation of exenatide or insulin treatment. In addition, the Modified Rankin Scale will assess functional status premorbid, and 3 months post-stroke. The mean change in stroke scale(s) from baseline will be compared between the two treatment arms. Blood samples will be collected at various time points throughout the duration of exenatide or insulin infusion $(0,24$, 48 and $72 \mathrm{~h}$ ), and analysed for circulating inflammatory markers and metabolic hormone levels. Safety and tolerability of exenatide therapy will be assessed through the incidence of adverse events, and the requirement for antiemetic therapy. A follow-up study visit will take place at 3 months postcompletion of exenatide or insulin therapy, where glycaemic control and neurological investigations will take place. The overall study design is highlighted in figure 1. Further information regarding collection and management of study data, safety reporting and post-trial care are included in the appendices and online supplementary material.

\section{Study end points}

The primary end point of this study is to determine the percentage of time that the participant's blood glucose levels remain within a target glucose range of 5-10 mmol/L, as determined by continuous glucose monitoring (CGM). In addition, the secondary end point of this study is the frequency and duration of hypoglycaemia in participants receiving exenatide versus insulin therapy. For acute management decisions, hypoglycaemia is defined as a capillary blood glucose level $<4 \mathrm{mmol} / \mathrm{L}$. In the analysis of the CGM data, an interstitial glucose level $<4 \mathrm{mmol} / \mathrm{L}$ will be used for the identification of hypoglycaemia that is unrecognised by finger-stick capillary glucose measurements.

A further secondary end point comprises the percentage of time in which the participant's blood glucose levels remain within the target range in the first $24 \mathrm{~h}$ after commencing exenatide treatment. The change in National Institutes of Health Stroke Scale from baseline, $24 \mathrm{~h}, 3$ days and 3 months post-study treatment initiation, and the change in Modified Rankin Scale at 3 months post-stroke will also be reported although it is not believed that this study is powered to provide a robust assessment of these end points.

In addition, the feasibility of intravenous exenatide administration in the setting of AIS will be assessed through the use of a questionnaire directed towards the relative difficulty of implementing an intravenous exenatide protocol in this setting versus intravenous insulin administration. Finally, the safety and tolerability of exenatide therapy in patients with AIS will be evaluated. All study end points are outlined in box 2 .

\section{Statistical methods and sample size Sample size}

As this is a pilot study looking at preliminary efficacy and safety of exenatide treatment in this population, the results will inform further clinical investigations and larger trial designs. The total number of enrolled patients will be 30 , with 15 in each arm. Based on historical records, we have estimated that recruitment of this sample size is achievable within the study period of 18 months, and will allow for testing the feasibility of implementing exenatide therapy in this setting, and enable monitoring of adverse events. In addition, with a sample size of 30 patients, we calculated $80 \%$ power to observe a difference between the treatment arms in the 
Screening of patients presenting with acute ischaemic stroke
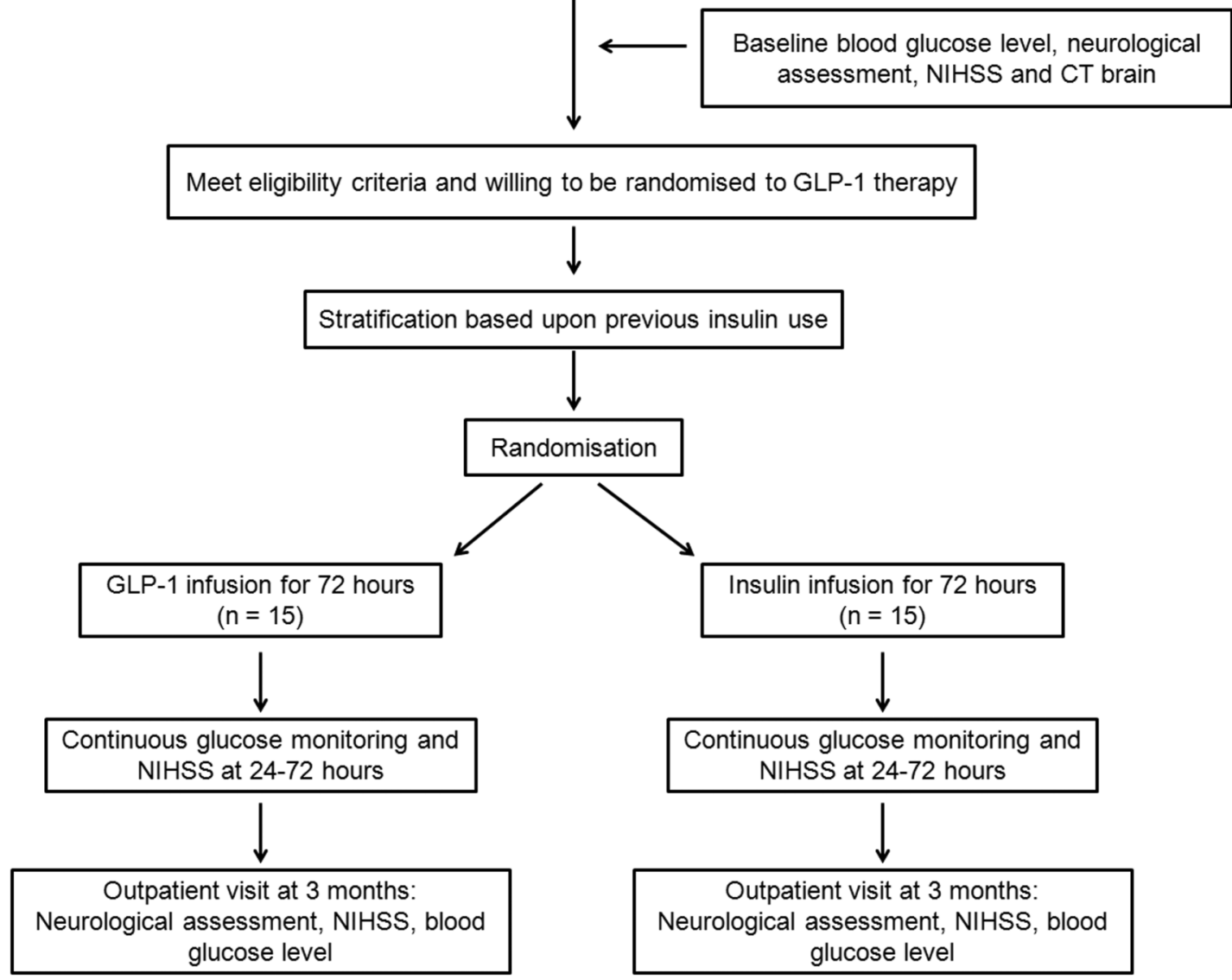

Figure 1 Study design flow chart. Glp, Glucagon-like peptide 1; NIHSS, National Institutes of Health Stroke Scale.

incidence of hypoglycaemia, of $5 \%$ in one group and $55 \%$ in the other group at $\alpha=0.1$.

\begin{tabular}{|c|c|c|c|}
\hline $\begin{array}{l}\text { Incidence } \\
\text { of } \\
\text { hypoglycaemia } \\
\text { in exenatide } \\
\text { arm } \\
\text { (group 1) (\%) }\end{array}$ & $\begin{array}{l}\text { Incidence } \\
\text { of } \\
\text { hypoglycaemia } \\
\text { in insulin } \\
\text { arm } \\
\text { (group 2) (\%) }\end{array}$ & $\begin{array}{l}\text { Power } \\
\text { level } \\
\text { (\%) }\end{array}$ & Significance \\
\hline 5 & 55 & 80 & 0.1 \\
\hline 10 & 60 & 80 & 0.1 \\
\hline 30 & 80 & 80 & 0.1 \\
\hline 40 & 90 & 80 & 0.1 \\
\hline
\end{tabular}

Assuming an average of $60 \%$ (SD 15) time within the target blood glucose range in standard care, we would have $80 \%$ power to detect an average time within range of $80 \%$ in group 2 with $\alpha=0.1$.

\section{Interim analyses}

After 16 patients have been recruited to the study (approximately $50 \%$ of the total number of participants to be recruited) an interim analysis of the efficacy of exenatide at lowering blood glucose levels, as well as the safety of exenatide therapy in the study population will be carried out (by study researchers that are not directly involved in the day-to-day conduct of the study). The frequency of requirement of insulin rescue therapy in the exenatide treatment arm will also be determined.

\section{Analysis plan}

The study results will be analysed using the intention-to-treat approach. The primary efficacy end point of the percentage time that patients' blood glucose levels are within the target range in the exenatide version insulin treatment arms will be evaluated using the Student $\mathrm{t}$ test and the Mann-Whitney $\mathrm{U}$ test. The incidence of hypoglycaemic events in each of the treatment arms will be compared using Fisher's exact test. Subgroup analysis includes stratifying the results based on patients' previous use of insulin, to determine whether this impacts on the efficacy of exenatide at lowering blood glucose levels.

\section{Ethics and dissemination}

\section{Dissemination}

The study results will be communicated through abstract submission and presentation at national and 


\section{Box 2 Study end points}

Primary end point

- The percentage of time in which participants' blood glucose levels remain within the target range of $5-10 \mathrm{mmol} / \mathrm{L}$.

Secondary end points

- Frequency and duration of hypoglycaemia in participants receiving exenatide vs insulin therapy. Hypoglycaemia is defined as a capillary blood glucose level/interstitial glucose level $<4 \mathrm{mmol} / \mathrm{L}$.

- The percentage of time in which participant's blood glucose levels remain within the target range $(5-10 \mathrm{mmol} / \mathrm{L})$ in the first $24 \mathrm{~h}$ after commencing exenatide treatment.

Other end points

- Change in National Institutes of Health Stroke Scale from baseline at $24 \mathrm{~h}, 3$ days and 3 months post-stroke.

- Modified Rankin Scale rating at 3 months.

- Frequency of low blood sugars $(4-5 \mathrm{mmol} / \mathrm{L})$ in exenatide vs insulin treatment arms.

- Feasibility of exenatide administration in the setting of acute ischaemic stroke.

- Safety and tolerability of exenatide therapy in acute ischaemic stroke.

international conferences, and submitted for publication in peer-reviewed scientific and medical journals.

\section{DISCUSSION}

The use of exenatide as an antihyperglycaemic agent in AIS has the potential to effectively lower blood glucose levels while reducing the incidence of hypoglycaemia. GLP-1 analogues readily reduce blood glucose levels in a glucose-dependent manner. Thus, exenatide therapy may give rise to improved outcomes for patients after acute stroke, as both hyperglycaemia and hypoglycaemia during stroke is associated with worse functional outcomes. We expect the results of this pilot study to show that exenatide is comparable to insulin at providing effective glycaemic control during AIS and, further, results in a lower rate of hypoglycaemic events and reduced glycaemic variability. Preliminary studies have shown that administration of GLP-1 gives rise to neuroprotective effects, in addition to reducing blood glucose levels. These neuroprotective effects include reduced inflammation and oxidative stress levels and decreased neuronal cell apoptosis. ${ }^{32}$ Thus, a further benefit in neurological outcomes for patients treated with exenatide may be observed, although this trial is not powered to examine functional end points. Overall, the results of this pilot study may provide promising preliminary data to support the use of exenatide at maintaining normoglycaemia in AIS, thereby improving outcomes for patients post-stroke.

\section{Author affiliations}

${ }^{1}$ Department of Diabetes, Endocrinology and Metabolism, Royal North Shore Hospital, Sydney, New South Wales, Australia

${ }^{2}$ University of Sydney, Northern Clinical School, Sydney, New South Wales, Australia
${ }^{3}$ Kolling Institute of Medical Research, Royal North Shore Hospital, St Leonards, New South Wales, Australia

${ }^{4}$ Department of Neurology, Royal North Shore Hospital, Sydney, New South Wales, Australia

Acknowledgements The authors thank Jillian Patterson of the Biostatistics Department, University of Sydney, for assistance with statistical methods.

Contributors RTM contributed towards the design of the study and drafted the initial protocol. SLH contributed to conception and design of the study, particularly the glycaemic control and glycaemia-based study assessments. GRF is the study leader and made a substantial contribution towards the initial conception and overall design of the study. MP, SD, GKH and MK contributed towards study design, specifically the neurological components of the trial. All authors were involved in finalising the study design and protocol. Further, all authors contributed to preparation of the manuscript and approved the final version of the manuscript.

\section{Competing interests None declared.}

Ethics approval The study protocol was approved by the local institutional review board (Northern Sydney Local Health District Human Research Ethics Committee; Reference No. RESP/14/95), and will be carried out in accordance with the principals of Good Clinical Practice, and the Declaration of Helsinki.

Provenance and peer review Not commissioned; externally peer reviewed.

Open Access This is an Open Access article distributed in accordance with the Creative Commons Attribution Non Commercial (CC BY-NC 4.0) license, which permits others to distribute, remix, adapt, build upon this work noncommercially, and license their derivative works on different terms, provided the original work is properly cited and the use is non-commercial. See: http:// creativecommons.org/licenses/by-nc/4.0/

\section{REFERENCES}

1. Lindsberg PJ, Roine RO. Hyperglycemia in acute stroke. Stroke 2004;35:363-4.

2. Baird TA, Parsons MW, Phan T, et al. Persistent poststroke hyperglycemia is independently associated with infarct expansion and worse clinical outcome. Stroke 2003;34:2208-14.

3. Bruno A, Biller J, Adams HP Jr, et al. Acute blood glucose level and outcome from ischemic stroke. Trial of ORG 10172 in Acute Stroke Treatment (TOAST) Investigators. Neurology 1999;52:280-4.

4. Capes SE, Hunt D, Malmberg K, et al. Stress hyperglycemia and prognosis of stroke in nondiabetic and diabetic patients: a systematic overview. Stroke 2001;32:2426-32.

5. Parsons MW, Barber PA, Desmond PM, et al. Acute hyperglycemia adversely affects stroke outcome: a magnetic resonance imaging and spectroscopy study. Ann Neurol 2002;52:20-8.

6. Kruyt ND, Biessels GJ, Devries JH, et al. Hyperglycemia in acute ischemic stroke: pathophysiology and clinical management. Nat Rev Neurol 2010;6:145-55.

7. O'Neill PA, Davies I, Fullerton KJ, et al. Stress hormone and blood glucose response following acute stroke in the elderly. Stroke 1991;22:842-7.

8. European Stroke Organisation Executive C, Committee ESOW Guidelines for management of ischaemic stroke and transient ischaemic attack 2008. Cerebrovasc Dis 2008;25:457-507.

9. Jauch EC, Saver JL, Adams HP Jr, et al. Guidelines for the early management of patients with acute ischemic stroke: a guideline for healthcare professionals from the American Heart Association/ American Stroke Association. Stroke 2013;44:870-947.

10. Gentile NT, Seftchick MW, Huynh T, et al. Decreased mortality by normalizing blood glucose after acute ischemic stroke. Acad Emerg Med 2006;13:174-80.

11. Staszewski J, Brodacki B, Kotowicz J, et al. Intravenous insulin therapy in the maintenance of strict glycemic control in nondiabetic acute stroke patients with mild hyperglycemia. J Stroke Cerebrovasc Dis 2011;20:150-4.

12. Hsu PF, Sung SH, Cheng HM, et al. Association of clinical symptomatic hypoglycemia with cardiovascular events and total mortality in type 2 diabetes: a nationwide population-based study. Diabetes Care 2013;36:894-900.

13. Preiser JC, Devos $P$, Ruiz-Santana S, et al. A prospective randomised multi-centre controlled trial on tight glucose control by intensive insulin therapy in adult intensive care units: the Glucontrol study. Intensive Care Med 2009;35:1738-48. 
14. Garg R, Hurwitz S, Turchin A, et al. Hypoglycemia, with or without insulin therapy, is associated with increased mortality among hospitalized patients. Diabetes Care 2013;36:1107-10.

15. Finfer S, Chittock DR, Su SY, et al. NICE-SUGAR Study Investigators. Intensive versus conventional glucose control in critically ill patients. N Engl J Med 2009;360:1283-97.

16. Bellolio MF, Gilmore RM, Stead LG. Insulin for glycaemic control in acute ischaemic stroke. Cochrane Database Syst Rev 2011;(9): CD005346.

17. Gray CS, Hildreth AJ, Sandercock PA, et al. Glucose-potassiuminsulin infusions in the management of post-stroke hyperglycaemia: the UK Glucose Insulin in Stroke Trial (GIST-UK). Lancet Neurol 2007:6:397-406.

18. Ntaios G, Egli M, Faouzi M, et al. J-shaped association between serum glucose and functional outcome in acute ischemic stroke. Stroke 2010;41:2366-70.

19. Drucker DJ. The biology of incretin hormones. Cell Metab 2006;3:153-65.

20. Meloni AR, DeYoung MB, Lowe C, et al. GLP-1 receptor activated insulin secretion from pancreatic $\beta$-cells: mechanism and glucose dependence. Diabetes Obes Metab 2013;15:15-27.

21. Darsalia $\mathrm{V}$, Mansouri $\mathrm{S}$, Ortsäter $\mathrm{H}$, et al. Glucagon-like peptide-1 receptor activation reduces ischaemic brain damage following stroke in Type 2 diabetic rats. Clin Sci (Lond) 2012;122:473-83.

22. Teramoto S, Miyamoto N, Yatomi K, et al. Exendin-4, a glucagon-like peptide-1 receptor agonist, provides neuroprotection in mice transient focal cerebral ischemia. J Cereb Blood Flow Metab 2011;31:1696-705

23. Briyal S, Gulati K, Gulati A. Repeated administration of exendin-4 reduces focal cerebral ischemia-induced infarction in rats. Brain Res 2012;1427:23-34.
24. Li Y, Perry T, Kindy MS, et al. GLP-1 receptor stimulation preserves primary cortical and dopaminergic neurons in cellular and rodent models of stroke and Parkinsonism. Proc Nati Acad Sci USA 2009;106:1285-90.

25. Daly SC, Chemmanam T, Loh PS, et al. Exenatide in acute ischemic stroke. Int J Stroke 2013;8:E44.

26. Gejl M, Lerche S, Egefjord L, et al. Glucagon-like peptide-1 (GLP-1) raises blood-brain glucose transfer capacity and hexokinase activity in human brain. Front Neuroenerget 2013;5:2.

27. Scholte M, Timmers L, Bernink FJ, et al. Effect of additional treatment with Exenatide in patients with an Acute Myocardial Infarction (EXAMI): study protocol for a randomized controlled trial. Trials 2011;12:240.

28. Lønborg J, Vejlstrup N, Kelbæk H, et al. Exenatide reduces reperfusion injury in patients with ST-segment elevation myocardial infarction. Eur Heart J 2012;33:1491-9.

29. Degn KB, Brock B, Juhl CB, et al. Effect of intravenous infusion of exenatide (synthetic exendin-4) on glucose-dependent insulin secretion and counter regulation during hypoglycemia. Diabetes 2004:53:2397-403.

30. Fehse F, Trautmann M, Holst JJ, et al. Exenatide augments firstand second-phase insulin secretion in response to intravenous glucose in subjects with type 2 diabetes. J Clin Endocrinol Metab 2005;90:5991-7.

31. Nauck MA, Baranov O, Ritzel RA, et al. Do current incretin mimetics exploit the full therapeutic potential inherent in GLP-1 receptor stimulation? Diabetologia 2013;56:1878-83.

32. McGrath R, Glastras S, Hocking S, et al. Central functions of glucagon-like peptide-1: roles in energy regulation and neuroprotection. J Steroids Horm Sci 2015;6:152. doi:10.4172/ 2157-7536.1000.152 